\title{
Electrical and Optical Characteristics of InAs Junction Lasers
}

\author{
NAVIN PATEL AND AMNON YARIV, FELLOW, IEEE
}

\begin{abstract}
Electrical and light emission characteristics of pulsed InAs $\mathrm{p}-\mathrm{n}$ junction lasers, prepared by diffusing $\mathrm{Zn}$ into $\mathrm{Sn}$-doped n-type crystals with a substrate concentration of $10^{18} \mathrm{~cm}^{-3}$, were studied at $4.2,20.4$, and $77^{\circ} \mathrm{K}$. Data of variation of capacitance with bias showed these to be abrupt junctions. At injection currents below threshold, the temperature independence of the slopes of the $I-V$ characteristics and the observed variation of the integrated light intensity with current suggest the injection mechanism be photon-assisted tunneling. The peak of the spontaneous radiation shifts with the applied voltage in the expected manner. The timeresolved laser spectra are seen to shift to longer wavelengths during pulsed operation of the laser. The line shift results from joule heating of the junction, and follows the variation of bandgap with temperature. From a plot of threshold current density as a function of reciprocal length gain and loss factors have been calculated. An independent value of the gain factor was obtained from the observed variation with current of the superradiantly narrowed linewidth from a nonlasing structure. Mode-confinement studies indicate that the mode confinement is due to the optical gain in the junction region.
\end{abstract}

\section{INTRODUCTION}

【 ASER action in InAs junctions was first reported by Meingailis [1] who also reported effects of magnetic fields on the emission from these junctions, efficiency measurements, results on optically pumped InAs lasers, and the various states involved in optical transitions in both n- and p-type materials [2]-[4]. In this paper we describe results of experiments conducted with $p-n$ junction lasers in InAs, which are aimed at understanding the various physical processes obtaining in these pulsed junctions during spontaneous and stimulated radiative recombination.

The spontaneous emission at low current levels is investigated in parallel with the electrical characteristics. Several aspects of the emission at high current levels that are investigated include mode confinement in the diode cavity, variation of threshold current density with cavity length, superradiant narrowing, and laser line shift due to joule heating of the junction.

\section{Device Preparation}

All the p-n junctions were made by vapor diffusing $\mathrm{Zn}$ into an n-type substrate, which was Sn doped with a donor concentration of $10^{18} \mathrm{~cm}^{-3}$. Diffusions were made into polished (100) and (111) faces as well as into cleaved

Manuscript received February 24, 1970. This paper was presented at the IEEE Semiconductor Laser Conference, Mexico City, Mexico, December 1969.

The authors are with the California Institute of Technology, Pasadena, Calif.
(110) faces. Junction depths ranged from 10 to $25 \mu$. Ohmic contacts were made by using an evaporated $\mathrm{Zn}-\mathrm{Au}$ alloy for the $\mathrm{p}$ side and an $\mathrm{In}-\mathrm{Sn}-\mathrm{Pb}$ alloy solder for the $\mathrm{n}$ side. The diodes were mounted on transistor headers, which were directly immersed in a cryogenic Dewar.

\section{Recombination by Tunneling at Low: Injection}

Numerous studies have been made in narrow degenerate p-n junctions in GaAs [5]-[8], on the nature of injection mechanisms resulting in radiative recombination at low levels of injection before thermal injection sets in. The fact that the current-voltage characteristic is independent of temperature in this region indicates that thermal injection is not important. A characteristic feature is that energy at the peak of the emitted spectrum approximately equals the applied voltage and shifts both in position and intensity with the applied voltage.

To explain this phenomenon Leite et al. [5], [6], used the photon-assisted tunneling model, suggested earlier by Pankove [9], in which electrons and holes near Fermi levels in their respective bands can, because of the junction field, tunnel across the junction and recombine radiatively.

This kind of tunneling has also been reported in $\mathrm{PbTe}$ junctions at low temperatures [10].

The model predicts an exponential dependence of the radiation intensity on the applied voltage, i.e., $I \sim e^{S r V}$, and the magnitude of the logarithmic slope is given for the case of an abrupt junction by

$$
S_{T}=\left(\frac{e}{2}\right)^{3 / 2} \alpha W_{1}
$$

with

$$
\alpha=\frac{\pi m_{T}^{1 / 2}}{2 e \hbar},
$$

$m_{T}$ being the reduced effective tunneling mass and $W_{1}$ is the width constant that appears in the relation

$$
W=W_{1}\left(V_{i}-V\right)^{1 / 2}
$$

between the junction width $W$, the applied bias $V$, and the built in junction voltage $V_{i}$.

Another feature of photon-assisted tunneling is that the frequency $\nu_{P}$ of the peak of the output radiation is related to the applied bias by $h \nu_{p} \cong \mathrm{eV}$.

Measurements on the variation of junction capacitance with bias showed that the junctions were abrupt, with zero bias depletion widths ranging from about 400 to $2000 \AA$.

Fig. 1 shows the current-voltage characteristics at 77, 20.4 , and $4.2^{\circ} \mathrm{K}$ together with the shift in the peak $h \nu_{p}$ 


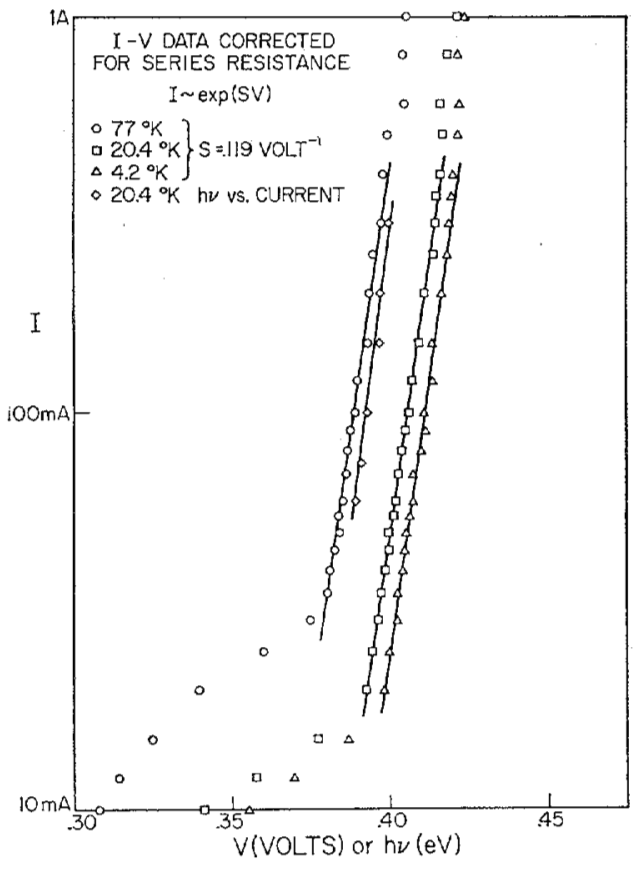

Fig. 1. Current $I$ as a function of bias $V$ and $h \nu_{\text {peak. }}$.

as a function of the current at $20.4^{\circ} \mathrm{K}$. Fig. 2 shows the total integrated light output as a function of the injection current at these temperatures in a typical diode. From the capacitance-voltage data for this diode, $W_{1}$ was found to be $1.64 \times 10^{-5} \mathrm{~cm} / \mathrm{V}^{1 / 2}$.

The observed temperature independent slope is

$$
S_{T^{\prime}}=119 / \text { volt. }
$$

The calculated value substituting the observed $W_{1}$ into expression (1) is

$$
S_{T^{\prime}}=104 / \text { volt. }
$$

Similar agreement of the observed slopes with the values predicted by the photon-assisted tunneling model was found for diodes with different junction widths.

\section{Heating Effects and Time-Resolved Spectra}

When injection lasers are driven by current pulses, the temperature rise during the pulse causes transient changes in the observed spectra. All pulsed injection lasers exhibit "mode jumping" to longer wavelengths during the time interval when the laser is pumped, particularly under conditions of high-peak current. As the temperature of a junction laser is changed two kinds of wavelength shifts are observed. First, the peak of the broad emission shifts, approximately at the same rate as the energy gap. In addition, there is a shift of individual modes with temperature, usually at a smaller rate. Thus, as the temperature is raised, a particular mode will change its wavelength continuously, but will have its amplitude diminish and become unobservable when it no longer falls within the spontaneous emission envelope. Both these effects have been extensively studied in GaAs junction lasers [11]-[14].

Several sources of heat generation can cause a junctiontemperature rise: 1) joule power dissipation in the semi-

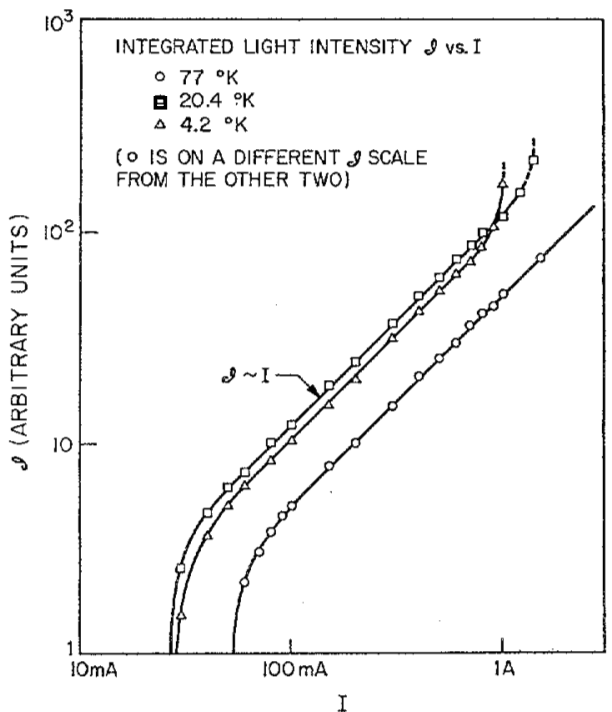

Fig. 2. Integrated light intensity as a function of current $I$.

conductor bulk region and at the contacts; 2) reabsorption of the spontaneous and stimulated radiation; and 3) nonradiative recombination in the junction region.

We will show that in the InAs junction lasers used, the first effect predominates at moderate to high peak currents and that the shift of the peak photon energy of the emission follows the change of bandgap with temperature.

The laser (at $77^{\circ} \mathrm{K}$ ) is pulsed with square pulses at a repetition rate of $20 \mathrm{ps}$. The emitted radiation is spectrally resolved by passing it through a grating spectrometer and then detected with a high-speed (50-ns) detector $(\mathrm{Hg}-$ doped germanium operated at $20.4^{\circ} \mathrm{K}$ ).

The detector output is fed into a boxcar integrator with a gate width of $0.2 \mu \mathrm{s}$, and the de output from the boxcar drives a pen recorder. The spectral content of the light pulse at a particular time along the pulse is measured by choosing the gating time of the boxcar integrator to coincide with that time and then scanning with the spectrometer. In order to follow the shift of the peak of the broad emission, the resolution of the spectrometer is reduced so that neighboring modes merge into one another.

Fig. 3 shows typical data that illustrate the line shift in the time-resolved spectra for a current of 6.0 amperes. Fig. 4 shows the line shift as a function of time for various injection currents. It is seen that the line shift is linear with time and is proportional to the square of the current, indicating that the junction-temperature rise is due to joule heating. A linear relationship with current would have indicated reabsorption and/or nonradiative processes as the source of heating.

The shift is seen to follow the relation

$$
\Delta \lambda=k I^{2} t
$$

where for this particular diode the average value of the lineshift coefficient $k$ is found to be

$$
k=0.77 \AA / \mathrm{A}^{2}-\mu \mathrm{s} .
$$

The line-shift coefficient $k$ can be calculated from the 


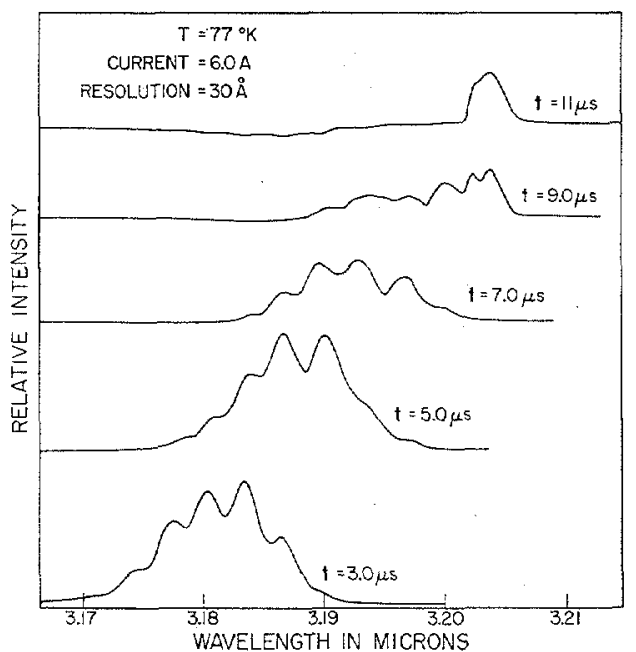

Fig. 3. Time-resolved spectra at several times $t$ during the applied pulse; $11.5-\mu$ s pulses were used.

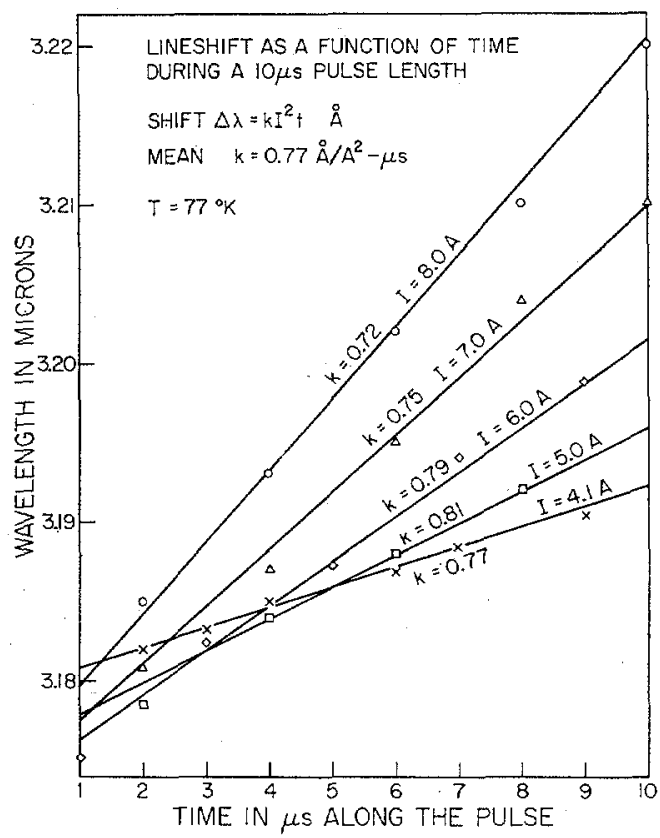

Fig. 4. Line shift as a function of time during a 10- $\mu$ s pulse length for several different current amplitudes.

device and material parameters. The joule heating power is $P_{j}=I^{2} R$ where $R$ is the series resistance of the diode. Neglecting cooling, the junction temperature rise is given by

$$
\Delta T(t)=\frac{P_{i} t}{c d v}
$$

where $e$ is the specific heat capacity of the material at $77^{\circ} \mathrm{K}, d$ is the density, and $v$ the volume of the diode.

If we assume that the laser-line temperature coefficient is identical to that for the bandgap $\left(-2.8 \times 10^{-4}\right)^{15}$, the laser-line shift is given by

$$
\delta E=-2.8 \times 10^{-4} \Delta T \mathrm{eV} .
$$

Writing this in terms of $\delta \lambda$ and substituting for $\Delta T$ form
(5) we get

$$
\delta \lambda=0.74 I^{2} t
$$

where $t$ is in $\mu \mathrm{s}, I$ in amperes, and $\delta \lambda$ in $\AA$.

We see that the calculated line-shift coefficient agrees quite well the measured value.

It can be demonstrated that joule heating can be expected to dominate over the other two processes for the range of currents used in this experiment. Compared with the joule heating power $P_{i}=I^{2} R$, the rate of heating by the other two processes is $P_{n}=\left(1-\eta_{\text {ext }}\right) I V_{o}$. For this diode, $R=0.33 \mathrm{ohm}, \eta_{\mathrm{ext}} \approx 0.1$, and also $V_{v} \simeq 0.4$ volt. Thus we see that for the currents used (4-8 amperes) $P_{i}$ will dominate over $P_{n}$.

\section{Gain and Loss Factors at $20.4^{\circ} \mathrm{K}$}

A method for determining the laser gain and loss factors consists of measuring the threshold current density for a series of lasers with different lengths, but otherwise identical structures [16], [17]. A comparison of different diodes is possible only if they are of uniform quality. This requires a uniform diffusion front as well as a uniform current distribution. The latter was achieved by using large area contacts made by covering the whole surface area on the $p$ side with a zinc-gold film. To assure uniform diffusion fronts in a given group of diodes, batches of diodes of different lengths were made from small sections of the larger wafers into which the $\mathrm{Zn}$ diffusion was made.

Fig. 5 shows the threshold current density $J_{\text {th }}$ plotted as a function of $1 / l$ for two sets of diode samples. A reasonable linear fit can be made to the experimental points.

From the threshold relationship

$$
e^{\left[\gamma\left\langle J_{\mathrm{th}}\right)-\alpha \ell l\right.} R=1
$$

it follows that the exponential gain constant at threshold is given by

$$
\gamma\left(J_{\mathrm{tb}}\right)=1 / l \ln 1 / R+\alpha
$$

where $\alpha$ is the loss constant and $R$ is the reflectivity at the cleaved ends. A linear relationship between $J_{\text {th }}$ and $1 / l$ is obtained if the gain is proportional to the current density $[\gamma=J / \beta]:$

$$
J_{\mathrm{th}}=\beta[\alpha+1 / l \ln (1 / R)] .
$$

Using this relationship, the values of $\alpha$ and $\beta$ determined for the two sets of diodes are

$$
\begin{array}{ll}
\alpha=40 \mathrm{~cm}^{-1} & \beta=10 \mathrm{~A} \cdot \mathrm{cm}^{-1} \\
\alpha=40 \mathrm{~cm}^{-1} & \beta=7.5 \mathrm{~A} \cdot \mathrm{cm}^{-1} .
\end{array}
$$

An interesting side experiment was performed that also gave a value for the gain coefficient $\beta$ at $20.4^{\circ} \mathrm{K}$. Measurements were made on the variation with current of the superradiant line narrowing of the radiation from a nonlasing structure, in which one of the ends was cleaved at an angle to the other. (The cleavage was not perfect, but good enough to spoil the resonance of the cavity.) This diode was made from a different part of the wafer from 

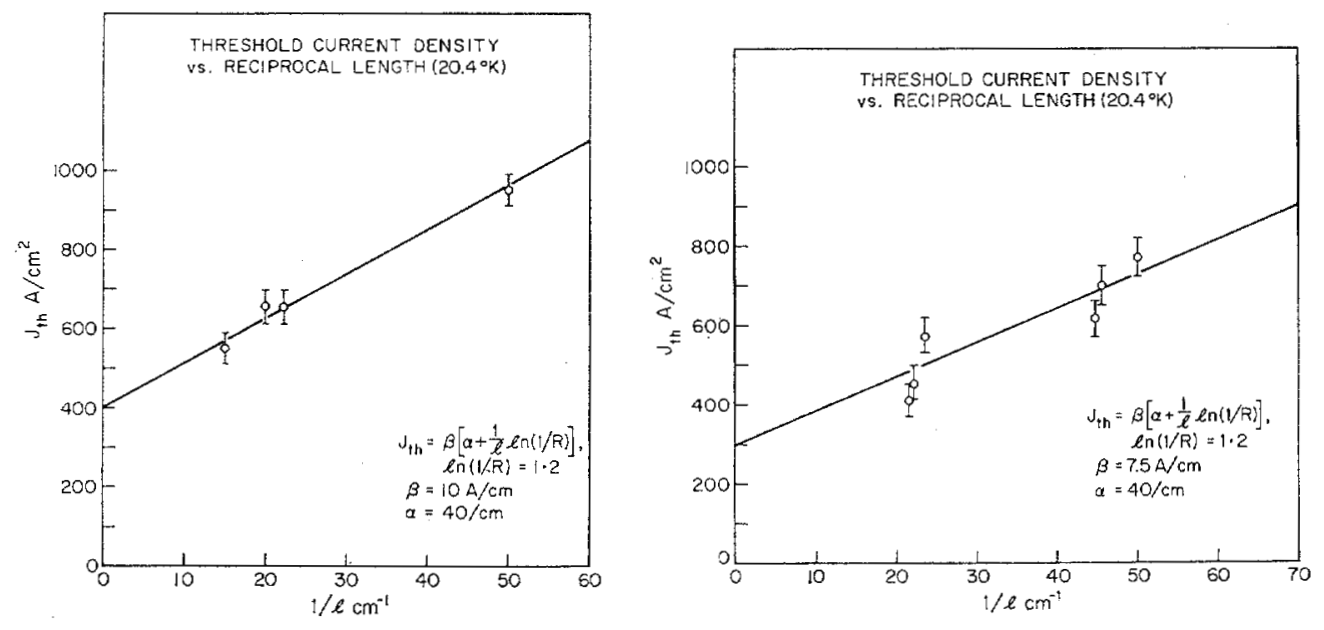

Fig. 5. Threshold current density $J_{\text {th }}$ as a function of reciprocal length $l$.

which samples of the first batch were made, and so a direct comparison of the values of $\beta$ was not possible.

It can be shown [18] that for injection currents such that gain is considerably larger than the loss

$$
\frac{\delta \nu}{\Delta \nu}=\left[\gamma\left(\nu_{0}\right) l\right]^{-1 / 2}
$$

where

$\Delta \nu=$ width of the spontaneous emission,

$\delta \nu=$ width of the gain-narrowed line,

$\gamma\left(\nu_{0}\right)=$ gain per unit length at line center and $l$ is the length through which the light is amplified.

Using $\gamma\left(\nu_{0}\right)=J / \beta$ we have

$$
\frac{\delta \nu}{\Delta \nu}=\left[\frac{J l}{\beta}\right]^{-1 / 2}
$$

From a plot of $\delta \nu / \Delta \nu$ against $I^{-1 / 2}$ a value of $\beta$ can be obtained. Fig. 6 shows such a plot for the diode described above. The value of $\beta$ obtained is

$$
\beta=16 \mathrm{~A} \cdot \mathrm{cm}^{-1} \text {. }
$$

\section{Mode Confinement}

In this section we describe an experiment in which the measured far-field diffraction pattern of a diode is Fourier transformed to obtain the field distribution at the junction, which is in excellent agreement with that expected using a simple dielectric waveguide model [19].

The model is a symmetrical three-layer structure (Fig. 7 ) in which the inner layer, with thickness $2 d$ and dielectric constant $\epsilon_{i}$ is sandwiched between two semi-infinite media of dielectric constant $\epsilon=\epsilon_{i} / K$, where $K$ is larger than unity. Solving Maxwell's equation for a TE-even mode, we get for the intensity variation across the junction,

$I(x)=A^{2} \cos ^{2} h x|x| \leq d$

$I(x)=A^{2} \cos ^{2} h d \exp [-2 p(|x|-d)] \quad|x| \geq d$

where $A$ is a constant and $h, p$, and $d$ are related to $K$ and the wave number $k$ by

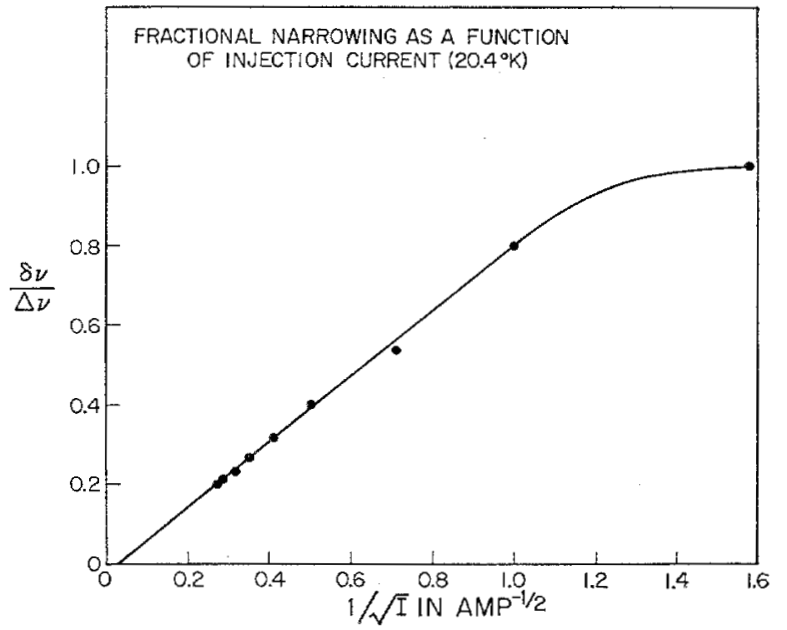

Fig. 6. Fractional narrowing of the spontaneous radiation as as function of injection current.

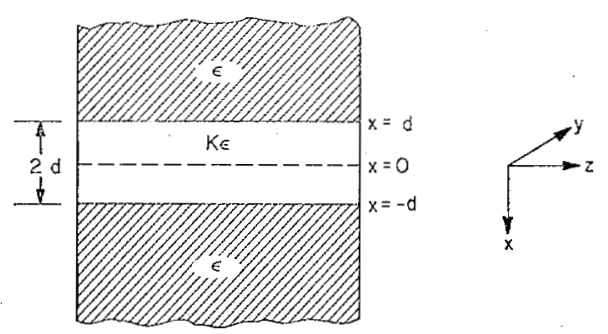

Fig. 7. Geometry of diode dielectric cavity.

$$
\begin{gathered}
p d=h d \tan h d \\
(p d)^{2}+(h d)^{2}=(K-1) k^{2} d^{2} .
\end{gathered}
$$

Using the notation shown in Fig. 8, the diffraction field $E_{y}\left(x^{\prime}\right)$ due to the source field $E_{y}(x)$ is of the form [20], [10]

$$
E_{y}\left(x^{\prime}\right) \sim \int_{p} \frac{E_{y}(x) e^{-(2 \pi i / \lambda)} S\left(x^{\prime} x\right)}{S\left(x^{\prime}, x\right)} d x
$$

where it is assumed that the phase of the field $E_{y}(x)$ is constant over the width $F$ of the emitting surface. For the case of far-field Fraunhofer diffraction this becomes 


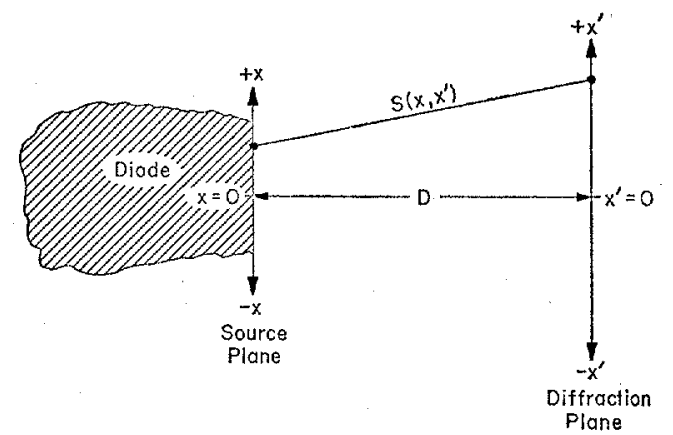

Fig. 8. Geometry for diffraction-field calculation.

$$
E_{y}\left(x^{\prime}\right) \sim \int_{p} E_{y}(x) e^{2 \pi i(x / \lambda)\left(x^{\prime} / D\right)} d x
$$

which is just the Fourier transform of $E_{y}$ in terms of the variables $x, x^{\prime}$. The inversion of (19) gives

$$
E_{y}(x) \sim \int_{F^{\prime}} E_{y}\left(x^{\prime}\right) e^{-2 \pi i(x / \lambda)\left(x^{\prime} / D\right)} d x^{\prime}
$$

where $F^{\prime}$ is the width of the far-field pattern.

Fig. 9 shows the measured $I\left(x^{\prime}\right)$ for junction $L_{26}$ when it was pulsed with a current 15 percent above threshold. Fig. 10 shows the Fourier tranformed $I(x)$ obtained from the data of Fig. 9, under the assumption of constant phase at the emitting surface. Also shown are curves for (15) with values of $h$ and $p$ chosen to fit the values obtained for $I(x)$.

The value of $d$ obtained by using the values of $h$ and $p$ in (16) is consistent with the one obtained directly from the plot of $I(x)$ as a function of $x$. The value of $d$ agrees with the calculated diffusion length of electrons in p-type material at $20.4^{\circ} \mathrm{K}$.

Substituting these observed values of $h, p$, and $d$ in (17) we obtain

$$
\frac{\delta \epsilon}{\epsilon}=1.1 \times 10^{-3} .
$$

One of the mechanisms put forward [21] to explain the physical origin of the observed $\delta \epsilon / \epsilon$ in GaAs junctions is that in which the mechanism of the change of dielectric constant is directly related to the gain characteristic of the medium. (The high gain characteristic needed for laser action determines the extent of the region of high intensity in the diode structure). The magnitude of the change in this case is given by

$$
\frac{\delta \epsilon}{\epsilon} \simeq \frac{\alpha_{t}}{2 k p d}
$$

where $\alpha_{t}$ is the total exponential intensity loss coefficient $\left(I \sim e^{-\alpha_{t} z}\right)$.

From the data of the previous section, $\alpha_{t}$ for this diode is $70 / \mathrm{cm}$. Substituting this in (22) we get

$$
\frac{\delta \epsilon}{\epsilon}=1.4 \times 10^{-3}
$$

in good agreement with (21).

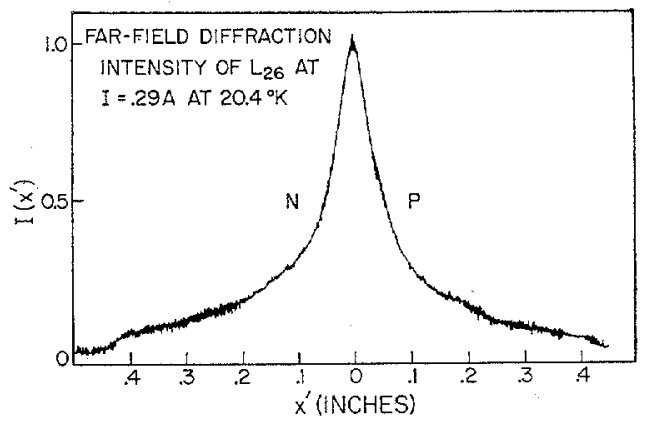

Fig. 9. Far-field radiation pattern in a plane perpendicular to the junction plane.

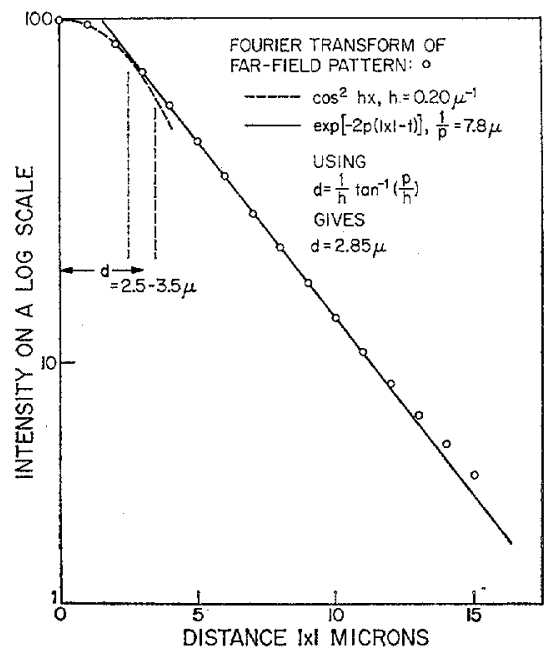

Fig. 10. Fourier transform of far-field pattern.

\section{SUMMARY}

The results of a number of experiments on the electrical and light emission characteristics of pulsed InAs $p-n$ junction lasers have been presented. At low current levels the current-voltage data exhibit a temperature independent $\left(4.2,20.4\right.$, and $77^{\circ} \mathrm{K}$ ) exponential variation of current with junction voltage. The current-voltage data together with the total light intensity data as a function of diode current indicate that photon-assisted tunneling is the dominant injection mechanism in this region. Time-resolved spectra show that when pulse operated InAs injection lasers show a laser line shift to longer wavelengths that is linear with time during the pulse length. The line shift has been shown to result from a junction temperature increase arising from joule power dissipation. Values for exponential gain and loss constants at $20.4^{\circ} \mathrm{K}$ were obtained from the observed variation of threshold current density as a function of reciprocal length of the laser cavity. The image-scan and far-field diffraction data indicate that the stimulated electromagnetic modes are confined to within approximately a diffusion length of the junction and that the mode confinement is due to the optical gain in the diode cavity.

\section{REFERENCES}

[1] I. Melngailis, "Maser action in InAs diodes," Appl. Phys. Letters, vol. 2, pp. 176-178, May 1963.

[2] I. Melngailis and R. H. Rediker, "Properties of InAs lasers," J. Appl. Phys., vol. 37, pp. 899-911, February 1966. 
[3] —-, "Magnetically tunable CW InAs Diode maser," Appl. Phys. Letters, vol. 2, pp. 202-204, June 1963.

[4] F. L. Galfener, I. Melngailis, G. W. Wright, and R. H. Rediker, "Magnetic properties of InAs diode electroluminescence," J. A ppl. Phys., vol. 36, pp. 1574-1579, May 1965.

[5] R. C. C. Leite, J. C. Sarace, D. H. Olson, B. G. Cohen, J. M. Whelan, and A. Yariv, "Injection mechanisms in GaAs diffused electroluminescent junctions," Phys. Rev., vol. 137, pp. A1583A1590, March 1965.

[6] R. J. Archer, R. C. C. Leite, A. Yariv, S. P. S. Porto, and J. M. Whelan, "Electron-hole and electron, impurity band tunneling in GaAs luminescent junctions," Phys. Rev. Letters, vol. 10, p. $483,1963$.

[7] G. C. Dousmanis, C. W. Mueller, and H. Nelson, "Effect of doping on frequency of stimulated and incoherent emission in GaAs diodes," Appl. Phys. Letters, vol. 3, pp. 133-135, October 1963.

[8] D. F. Nelson, M. Gershenzon, A. Ashkin, L. A. D'Asaro, and J. C. Sarace, "Band-filling model for GaAs injection luminescence," vol. 2, pp. 182-184, May 1963.

[9] J. I. Pankove, "Tunneling-assisted photon emission in GaAs p-n junctions," Phys. Rev. Letters, vol. 9, pp. 283-285, October 1962 .

[10] P. J. Zoutendyk, "Spontaneous and stimulated light emission due to radiative recombination in forward biased $\mathrm{PbTe} p-n$ junctions," Ph.D. dissertation, California Institute of Technology, Pasadena, Calif., May 1968.

[11] G. Burns and M. I. Nathan, " $P-N$ junction lasers," Proc. IEEE, vol. 52, pp. 770-794, 1964.
[12] C. H. Gooch, "Transient thermal effects in GaAs injection lasers," Phys. Letters, vol. 16, pp. 5-6, May 1965.

[13] J. D. Kingsley, and G. E. Fenner, "Stimulated emission from p-n junctions," Proc. Brd Internatl. Conf. on Quantum Electronics, vol. 2, pp. 1883-1890, 1964.

[14] T. Gonda, H. Junker, and M. F. Lamorte, "Time resolved spectral output of pulsed GaAs lasers," IEEE J. Quantum Electronics, vol. QE-1, pp. 159-163, July 1965.

[15] J. R. Dixon and J. M. Ellis, "Optical properties of n-type InAs in the fundamental absorption edge region," Phys. Rev., vol. 123, pp. 1560-1566, September 1961.

[16] M. Philkun and H. Rupprecht, "A relation between the current density at threshold and the length of the Fabry-Perot type GaAs lasers," Proc. IEEE (Correspondence), vol. 51, pp. 12-13, September 1963.

[17] M. Philkun, H. Rupprecht, and S. Blum, "Effect of temperature on the stimulated emission from GaAs p-n junctions," SolidState Electron., vol. 7, pp. 905-909, 1964.

[18] A. Yariv and R. C. C. Leite, "Super radiant narrowing in fluorescence radiation of inverted populations," J. Appl. Phys., vol. 34, pp. 3410-3411, November 1963.

[19] A. Yariv and R. C. C. Leite, "Dielectric-waveguide mode of light propagation in p-n junctions," Appl. Phys. Letters, vol. 2, pp. 55-57, February 1963.

[20] J. B. Marion, Classical Electromagnetic Radiation. New York: Academic Press, 1965, ch. 12.

[21] R. C. C. Leite and A. Yariv, "On mode confinement in p-n junctions," Proc. IEEE, vol. 51, p. 1035, July 1963.

\title{
Excitons and Phonons in Processes of Laser Oscillation from II-VI Semiconductors
}

\author{
M. S. BRODIN, D. B. GOER, S. V. ZAKREVSKI, AND V. YA. REZNICHENKO
}

\begin{abstract}
The determination of the nature of optical laser transitions is one of the basic problems of semiconductor laser physics. It was shown earlier in certain mixed $\mathrm{CdS}_{x}-\mathrm{CdSe} \mathrm{e}_{1 \rightarrow x}$ crystals that in the case of intense dipole-allowed exciton absorption, the indirect exciton transition provides the principal channel for laser action using free excitons with the assistance of optical phonons at relatively high temperatures and using bound excitons at relatively low temperatures. In the present work new evidence is reported on the above $\mathrm{CdS}_{x}-\mathrm{CdSe}_{1-x}$ crystals as well as on other II-VI compounds $\left(\mathrm{ZnS}_{x}-\mathrm{CdS}_{1-x}\right.$ and $\left.\mathrm{ZnSe}-\mathrm{CdSe}\right)$, enabling conclusions about the characteristics of the oscillations produced by the complex phonon spectra of mixed crystals.

Lasing of the above crystals was produced by optical two-photon pumping using a ruby laser. Experimental results showed that with two-photon pumping involving excitons in crystals of $\mathrm{CdS}$, CdS-CdSe, ZnS-CdS, and $\mathrm{ZnSe}-\mathrm{CdSe}$, lasing can be obtained at
\end{abstract}

Manuscript received November 15, 1969. This paper was presented at the IEEE Semiconductor Laser Conference, Mexico City, Mexico, December 1969 .

The authors are with the Institute of Physics, Academy of Sciences of the Ukranian SSR, Kiev, USSR. temperatures no higher than $120-140^{\circ} \mathrm{K}$. When the temperature is not too low ( $T \geq 60^{\circ} \mathrm{K}$ ), the laser action always preceeds by means of free excitons interacting with LO phonons. At lower temperatures than $50^{\circ} \mathrm{K}$ most of the crystals lase on bound excitons, with or without the involvement of optical phonons, depending on the spectral distribution of losses.

In two cases ( $\mathrm{CdS}$ and $\mathrm{ZnS}_{0.09}-\mathrm{CdS}_{0.91}$ ) at $\mathrm{T}<50^{\circ} \mathrm{K}$, the lasing occurred on transitions of the Auger recombination type involving excitions.

The mechanism of laser action is a strong function of temperature.

An important feature of mixed crystals is the dependence of the phonons involved on the composition of the samples. This dependence was studied in the CdS-CdSe crystals. These crystals have a complex phonon spectrum; they are characterized by two types of longitudinal optical phonons, $\mathrm{LO}_{1}$ and $\mathrm{LO}_{2}$, whose intensity ratio is dependent on the crystal composition.

For crystals in which one of the components is dominant at $77^{\circ} \mathrm{K}$, it is shown that the oscillation occurs via free excitons with either the $L 0_{1}$ or $L O_{2}$ longitudinal optical phonon more intense. For crystals with comparable concentrations of their components, both $\mathrm{LO}_{1}$ and $\mathrm{LO}_{2}$ phonons take part simultaneously in the lasing process (twophonon transitions). 